\title{
Enea, la Sibilla e Dante: primi appunti su un quaresimale virgiliano
}

Énée, la Sibylle et Dante : premières considérations sur un sermonnaire pour le Carême virgilien

Aeneas, Sybil, and Dante: Preliminary Notes on a Virgilian Lenten Sermon

Collection

\section{Pietro Delcorno}

\section{OpenEdition Journals}

Edizione digitale

URL: http://journals.openedition.org/cei/5706

DOI: $10.4000 /$ cei.5706

ISSN: 2260-779X

Editore

UGA Éditions/Université Grenoble Alpes

\section{Edizione cartacea}

ISBN: 978-2-37747-159-1

ISSN: 1770-9571

Notizia bibliografica digitale

Pietro Delcorno, «Enea, la Sibilla e Dante: primi appunti su un quaresimale virgiliano», Cahiers d'études italiennes [Online], 29 | 2019, online dal 30 septembre 2019, consultato il 27 mars 2021. URL: http:// journals.openedition.org/cei/5706 ; DOI: https://doi.org/10.4000/cei.5706

Questo documento è stato generato automaticamente il 27 mars 2021

(c) ELLUG 


\title{
Enea, la Sibilla e Dante: primi appunti su un quaresimale virgiliano
}

\author{
Énée, la Sibylle et Dante : premières considérations sur un sermonnaire pour le \\ Carême virgilien \\ Aeneas, Sybil, and Dante: Preliminary Notes on a Virgilian Lenten Sermon \\ Collection
}

Pietro Delcorno

In questa santa Quaresima ho intenzione di predicare alcune cose che saranno piacevoli da ascoltare e che, tuttavia, provocheranno dolore e paura. E questo perché voglio predicare l'inferno secondo Virgilio, il quale nel sesto libro

dell'Eneide racconta che Enea discese proprio all'inferno e lì vide le orrende e stupefacenti pene dei peccatori ${ }^{1}$.

1 Il folgorante annuncio apre il sermone del Mercoledì delle Ceneri di un quaresimale conservato in forma incompleta in un manoscritto di inizio Quattrocento. Sulle soglie della Quaresima, l'anonimo predicatore - probabilmente un frate minore come lascia supporre un riferimento alle stimmate di Francesco - indica nell'Eneide la chiave di volta per strutturare un impegnativo ciclo omiletico, capace di condurre i suoi ascoltatori verso la Pasqua, e di supportare lo sviluppo didattico dei sermoni attraverso un racconto avvincente e spaventoso. Nelle intenzioni del predicatore, si facilitava così l'assimilazione dei contenuti teologici proposti, favorendo inoltre la partecipazione dei fedeli.

2 La Quaresima era diventata, a partire dal secondo Duecento, il tempo liturgico privilegiato per una predicazione quotidiana, intesa come forma intensificata di istruzione religiosa, capace di presentare agli ascoltatori in modo chiaro e sistematico 
gli elementi portanti della dottrina cristiana ${ }^{2}$. Il ritmo intensificato della predicazione quaresimale rappresentava un'occasione preziosa - ma anche una sfida. Si trattava infatti di gestire un ampio ciclo di sermoni, conquistando l'attenzione e la partecipazione degli ascoltatori. Proprio la necessità di rispondere a tali sfide spinse progressivamente i predicatori - o almeno, alcuni di essi - a sperimentare forme innovative nella costruzione dei propri quaresimali. Tali cicli potevano essere costruiti come macro-racconti o in forme semi-drammatiche, utili a vivacizzare e a legare insieme i sermoni attraverso una cornice narrativa sviluppata giorno per giorno, proponendo una storia nella quale gli ascoltatori erano sovente chiamati - implicitamente o esplicitamente - a identificarsi ${ }^{3}$.

3 Il sermonario al centro di questo contributo rappresenta un precoce esempio di tale strategia comunicativa. Il racconto dell'Eneide costituisce il suo filo conduttore: ogni sermone inizia con la sintesi - in alcuni casi, una vera e propria parafrasi ${ }^{4}-$ di una parte del libro VI dell'Eneide e con la citazione di alcuni suoi versi, spesso abbinati - come vedremo - a passi della Commedia di Dante. Il racconto di Virgilio veniva poi interpretato allegoricamente, in forma più o meno elaborata, prima di passare a un tema dottrinale ricavato dalla pericope evangelica del giorno. Il sermonario si presenta così come un'eccezionale forma di ricezione, appropriazione e 'trasformazione' del poema di Virgilio ${ }^{5}$. L'utilizzo dei classici va, infatti, in questo caso molto al di là di un'occasionale citazione o dell'uso allegorico di un singolo episodio ${ }^{6}$. Portando a un livello del tutto nuovo il riferimento a Virgilio nella predicazione dell'aldilà cristiano ${ }^{7}$, agli ascoltatori era presentata un'Eneide infernale, didattica, ad usum pulpiti - fossero essi litterati, già familiari con il poema, o idiotes messi a contatto con questo testo forse per la prima volta ${ }^{8}$.

\section{Il manoscritto e il copista}

4 Il testo di quello che potremmo definire Quaresimale virgiliano o Quaresimale di Enea è attestato, in forma incompleta, in Assisi, Biblioteca del Sacro Convento, Fondo Antico,

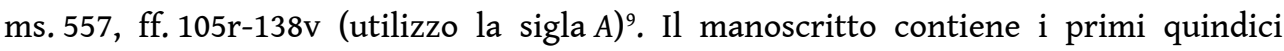
sermoni del ciclo. A metà di f. $136 \mathrm{v}$ viene copiato il thema del sedicesimo sermone, lasciando vuoti il resto della pagina e le ultime due carte del quaderno (ff. 137r-138v), segno evidente che per qualche ragione il copista interruppe il suo lavoro.

5 L'unico breve rimando a questo testo lo si deve alla descrizione del codice fornita da Cesare Cenci nel catalogo dei manoscritti della Biblioteca del Sacro Convento. Cenci riporta l'incipit del primo sermone, indica lo stato incompleto del quaresimale e segnala la presenza di citazioni dantesche, stranamente senza menzionare l'uso preponderante dell'Eneide ${ }^{10}$. Al medesimo studioso si deve l'individuazione del frate minore Filippo d'Assisi ( $\dagger$ c. 1435) come probabile copista del sermonario. La grafia di questa e altre sezioni del manoscritto è infatti la medesima di un altro codice del medesimo fondo, dove frate Filippo raccolse gli appunti delle prediche da lui tenute tra 1393 e 1397 in area umbra, mentre era lettore nel convento di Todi, come afferma all'inizio di un nuovo anno academico, probabilmente nel $1395^{11}$. Anche nel manoscritto 557, accanto a sermoni modello di altri autori ${ }^{12}$, Filippo d'Assisi raccolse gli appunti di alcuni suoi sermoni, tra i quali due ternioni (ff. 175r-186v) che contengono due brevi cicli di prediche tenute tra Avvento e tempo natalizio, uno a Rimini e l'altro ad Assisi. Il primo ciclo è sicuramente databile all'inverno 1414-1415, vista la presenza 
di una «Collatio facta pro domino Iacobo cardinali Utinensi» (ff. 178v-179r), predicata da Filippo a Rimini, dove era lettore ${ }^{13}$, nel tempo di Natale, a breve distanza dalla morte del cardinale Iacopino del Torso ${ }^{14}$. Il secondo ciclo si può probabilmente datare al periodo in cui il frate era custode del convento di Assisi (1418-1421) ${ }^{15}$. È ragionevole ipotizzare che anche il quadragesimale virgiliano sia stato copiato nello stesso torno d'anni, fissando così - in attesa di riscontri più precisi - alla fine del secondo decennio del Quattrocento il termine ante quem per la sua composizione.

\section{Seguendo Enea e la Sibilla}

6 L'affermazione con cui si apre il primo sermone - qui pubblicato integralmente in appendice - è seguita da una breve spiegazione su come il predicatore intenda procedere: ricondurrà (reducere) il racconto dell'inferno di Virgilio al Vangelo, facendone - dice - un piccolo sermone, per poi passare alla pericope evangelica del giorno, dalla quale promette di raccogliere un frutto (immagine ribadita in tutti i sermoni), utile a guidare gli ascoltatori verso la vita eterna. Lo schema bipartito permette di presentare anzitutto ciò che va fuggito e poi ciò che attrae verso la salvezza. Se nel concreto sviluppo dei sermoni la distinzione tra la funzione delle due parti non sarà in realtà così marcata, viene ribadito implicitamente come il racconto dell'Eneide - letto in chiave allegorico-morale - serva a impartire un salutare ammonimento, tale da allontanare dal peccato e predisporre al messaggio salvifico, secondo uno schema tipico delle visioni medievali dell'aldilà ${ }^{16}$.

7 Fornite le coordinate generali, nel primo sermone - particolarmente elaborato nella sua costruzione - si racconta dell'arrivo di Enea al tempio di Apollo e dell'invio di Acate, suo compagno, a chiamare la Sibilla, la quale, dopo avere rimproverato Enea intento a contemplare le immagini all'esterno del tempio, istruisce l'eroe troiano sul sacrificio da offrire prima di essere introdotto nell'antro dove gli verrà dato il responso richiesto $^{17}$. I versi citati al termine del racconto, Eneide VI, 36-41, ripetono l'esortazione della Sibilla a Enea perché non si soffermi a guardare le scene raffigurate sulla porta del tempio ma offra, invece, sette vitelli e sette agnelli in sacrificio. Il predicatore marca il passaggio all'interpretazione allegorica («istam particulam volo moraliter exponi»), ribadendo che seguirà questa modalità per raccordare l'Eneide al Vangelo anche i giorni successivi. Enea diventa così il simbolo di chiunque abbia perso tempo invano; ora invece, per Enea e gli ascoltatori, è il tempo di tornare a Dio (evidentemente, la Quaresima, come sottolinea subito la citazione di due inni liturgici) e la Sibilla - simbolo della divina sapienza - li invita a offrire a Dio un sacrificio adeguato. I sette agnelli simboleggiano infatti la mortificazione degli appetiti sensuali (il sette sta per i cinque sensi più la volontà e la sensualitas), mentre i sette giovenchi sono i sette peccati mortali, da 'immolare' a Dio scegliendo di vivere secondo le opposte virtù, così da essere introdotti nel tempio, simbolo della vita eterna.

Il racconto e la sua decodificazione in chiave morale formano, come annunciato, un «succinto sermone». L'interpretazione dell'episodio, se da un lato fa riferimento a elementi portanti della catechesi cristiana, non trova riscontri diretti nelle più diffuse letture allegoriche dell'Eneide. Né Servio, né Fulgenzio dedicano attenzione ai sette agnelli e sette tori ${ }^{18}$. Bernardo Silvestre, nella sua minuziosa analisi di Eneide VI, propone invece una doppia lettura: la prima interpreta i sette agnelli come le sette membra del corpo e i sette tori come i sette movimenti, da controllare e mortificare per 
potersi concentrare nello studio; la seconda identifica i tori come «septem virtutes carnem vexantes» (astinenza, moderazione, sobrietà, castità, frugalità, modestia, verecondia) mentre gli agnelli sono «septem simplicitatis et mansuetudinis virtutes» legate alla sfera relazionale (innocenza, amicizia, concordia, pietà, religione, affetto, umanità $)^{19}$. Si tratta di un'interpretazione solo lontanamente accostabile a quella proposta dal sermone, in cui il predicatore - nel caso conosca tale commento - sceglie un simbolismo diverso. Va poi notato come l'interpretazione serva a coinvolgere gli ascoltatori nella storia: Enea diventa simbolo di «chiunque tra noi», un noi inclusivo, utilizzato insistentemente, posizionando così predicatore e ascoltatori sullo stesso piano $^{20}-\mathrm{e}$ all'interno della vicenda raccontata.

\section{La porta del digiuno: Dante e due guardiani}

La transizione dall'Eneide alla pericope evangelica è costruita abilmente, sottolineando come Cristo stesso nel Vangelo del giorno esorti a compiere tale sacrificio e come, per «offrire e uccidere i sette tori e sette agnelli» menzionati dall'Eneide, non vi sia strumento migliore del digiuno - tema tradizionale del Mercoledì delle Ceneri e, giustamente, indicato come il fructum da raccogliere dalla pericope liturgica. La parte centrale del sermone analizza quattro aspetti: primo, come il digiuno sia stabilito da Dio e quale sia la specificità della Quaresima; secondo, come il digiuno venne imposto già nell'Eden e come, dopo la caduta, esso rappresenti «la porta» per un cammino salvifico; terzo, l'utilità del digiuno; quarto, il modo in cui bisogna digiunare. L'ultima parte in realtà non è marcata nel testo e si fonde con la recapitulatio dove, postillando il testo evangelico, si ribadiscono alcuni degli insegnamenti impartiti.

10 Là dove alcuni punti del sermone appaiono scarni schemi da arricchire, se necessario, nella performance orale ${ }^{21}$, particolarmente elaborata risulta la parte sul digiuno come porta, cioè inizio, del percorso penitenziale verso la salvezza. Se già il racconto dell'Eneide aveva presentato l'eroe troiano che, dopo aver compiuto i dovuti sacrifici, varcava la soglia per entrare nel tempio, il tema della porta è qui associato ai versi che nella Commedia descrivono l'ingresso alla montagna del purgatorio. La citazione dantesca unisce due momenti ravvicinati di Purgatorio IX: la visione della scala e del guardiano angelico (IX, 76-78); il gesto penitenziale del pellegrino e l'imposizione delle sette P sulla sua fronte (IX, 109-114). I versi di Dante sono presentati ma, a differenza di quelli di Virgilio, non vengono commentati ${ }^{22}$. Il collegamento non è però limitato all'immagine della porta. Gli ascoltatori minimamente familiari con il poema dantesco potevano cogliere facilmente nel simbolismo delle sette $P$ un riferimento ai sette peccati capitali, già menzionati nella prima parte della predica parlando del sacrificio da offrire a $\mathrm{Dio}^{23}$. Inoltre, l'idea di un guardiano di questa soglia è ripresa poco oltre nel sermone. Dopo aver sottolineato come lungo la storia il digiuno sia sempre stato utilizzato come strumento per invocare l'intervento divino (dagli antichi ai saraceni, da Adamo a Mosè ed Elia, fino a Francesco d'Assisi «volens Christi stigmatibus insigniri» ${ }^{24}$ ), si torna - attraverso un'icastica frase attribuita ad Ambrogio - alla definizione del digiuno come «porta paradisi».

11 Il concetto viene drammatizzato e reso 'visibile', dicendo che tale porta ha due guardiani, uno a destra e uno a sinistra, sdoppiando in un certo senso l'angelo «portiero» della Commedia. Il primo guardiano raffigura l'umiltà e ha in mano due cartigli, con adeguate citazioni bibliche che ricordano la natura mortale e l'essere terra 
e cenere dell'uomo ${ }^{25}$, tema immediatamente connesso al rito liturgico delle ceneri, dicendo che con tale guardia «iam fecimus legam» quando il sacerdote ha posto sul capo dei fedeli la cenere in forma di croce, a significare sia il ricordo della morte sia l'invito di Cristo a prendere la propria croce e seguirlo nel tempo di Quaresima. Il predicatore poneva così gli ascoltatori su una sorta di soglia virtuale: avendo già incontrato - anzi fatto lega - con la prima guardia, dovevano ora volgersi verso la seconda. Essa richiede loro una adesione interiore, simboleggiando la «recta intentio cordis ad Deum», la quale da un lato prega con le parole del Salmo 113 (Non nobis, Domine...) e dall'altro esorta chi entra - ovvero gli ascoltatori - a compiere ogni cosa nel nome di Gesù, insistendo, come al termine del sermone, su una sincera adesione al cammino penitenziale.

\section{Miseno alter Christus}

12 Il sermone analizzato chiarisce come il riferimento all'Eneide costituisca un tratto fondamentale del quaresimale, non solo nella costruzione della cornice narrativa, ma anche nel fornire la base - $\mathrm{o}$ il pretesto - per sviluppare un'interpretazione allegoricomorale che è già parte essenziale dell'insegnamento impartito dal pulpito. Se la modalità di costruire i sermoni e di leggere in chiave spirituale Virgilio richiederà uno studio più approfondito di quanto possibile proporre in questa sede, l'analisi di un altro passaggio permette di evidenziare come l'operazione compiuta risulti tutt'altro che banale.

13 Il racconto della morte di Miseno (Eneide VI, 149-174) occupa due sermoni. Nel primo, basato sull'annuncio da parte della Sibilla ad Enea che «in sua navi est quidam mortuus qui totam navim maculat fetore», illustra come sia necessario seppellire i vizi che macchiano la Chiesa (simboleggiata dalla nave) prima di accedere alla grazia divina (il ramo d'oro) che permetterà il viaggio nell'aldilà ${ }^{26}$. L'interpretazione morale sottolinea come la Chiesa sia macchiata «diversis vitiis [...] tam a laycis quam a clericis», ricollegando il tema della sua purificazione al Vangelo del giorno, la cacciata dei mercanti dal tempio (Matteo 21.12-17). Pur riconoscendo che ci sono due tipi di mercanti, il predicatore tratterà solo del primo - i laici - perché di coloro che hanno «curam pastoralem» bisogna parlare «cum sobrietate», come mostra Cristo che cacciò $i$ mercanti «cum flagellis», mentre rispose ai sacerdoti «cum mansuetudine» ${ }^{27}$. Stabilito questo, il sermone si incanala verso una discussione di etica economica, legate in particolare al tema dell'usura.

14 Il sermone successivo presenta la scoperta del cadavere di Miseno, definito «magnus tubator et probus in armis et bonus nauta», il quale «invidia fuit mortuus», venendo affogato da Tritone mentre era intento a suonare la tromba ${ }^{28}$. Il racconto è accompagnato da una doppia citazione dell'Eneide (VI, 161-163 e 171-174), seguita da un'interpretazione in cui, distaccandosi dalla lettura negativa proposta da Fulgenzio e Bernardo Silvestre (Miseno simbolo della lode effimera e della gloria mondana ${ }^{29}$ ), il predicatore avanza un'interpretazione cristologica, in cui gli elementi della descrizione dell'eroe troiano trovano riscontro nell'azione di Cristo.

Moraliter iste Misenus, licet non sit digna comparatio, tamen potest hic Christum significare, scilicet dei filium, qui licet primo fuerit comes magnus Ethoris, tamen comes nunc Henee. Ita Christus ante assumptione humanitatis fuit comes eternis patris quia cum patre unus in substantia. Nunc vero est comes Henee, id est est factus sotius per humanitatem omnium peccatorum. Sed huic nullus similis in 
tubando, in armis et in mari. Nam iste, scilicet Christus, sedavit mare turbatum, quia perambulavit eundem (cfr. Matteo 14.25). Unde de eo dicitur: Quis est hic quia venti et mare obediunt ei? (Matteo 8.27). Item fuit magnus preliator contra demones, quia iste est ille fortis armatus qui venit contra demones expellens eum (!) de domo et de habitattione sua (cfr. Luca 11.21-22). Unde Psalmus: Quis similis tui magnificus in sanctitate, terribilis atque laudabilis et faciens mirabilia? (Esodo 15.11). Iste fuit magnus quia iste est verbum dei terrens peccatores et animans bonos ad preliandi contra vitia et peccata. Et dum iste tubam caneret totumque mundum personaret, Trichon (!) tubator Neptuni ${ }^{30}$, id est scribe et pharisei, tubatores demonum inferni, contra Christum moti invidia, quia sua tuba predicationis contra eorum vitia clamabat, eum in mare mortis misserunt, id est cum amara morte eum interficerunt. Et hoc ostendit Christus in evangelio in exemplo Ione <qui> tribus diebus et tribus noctibus fuit in mare, unde dicit: Sicut fuit Ionas etc. (Matteo 12.40) ${ }^{31}$.

In questo modo, Miseno compagno prima di Ettore e poi di Enea diventa figura di Cristo che da compagno di Dio incarnandosi si è fatto compagno dell'uomo peccatore (Enea, con cui gli ascoltatori erano già stati invitati a identificarsi). Ugualmente, con una serie di rimandi biblici e un'abile costruzione a chiasmo, si dice che nessuno superò Cristo nel suonare la tromba, nel combattere, nel dominare il mare: egli infatti, camminò sulle acque, sconfisse i demoni e, in quanto Verbum Dei, spaventò i peccatori ed esortò alla lotta i compagni - come faceva Miseno. La consueta associazione tra predicazione e suono della tromba ${ }^{32}$ è ulteriormente sviluppata dicendo che, per fermare Cristo che ne denunciava i vizi, gli scribi e i farisei - i trombettieri infernali, identificati con Tritone - mossi dall'invidia decisero di affogarlo nel mare della morte, immagine questa prontamente ricollegata al Vangelo del giorno dove la vicenda di Giona prigioniero per tre giorni nel ventre del mostro marino era presentata da Gesù come figura della propria morte e resurrezione. Il sermone a questo punto passa alla parte più strettamente didattica, trattando dei sette spiriti che, entrando nell'uomo, lo rendono ostinato nel male (come gli avversari di Gesù nel racconto) e dei tre giorni trascorsi da Cristo nel sepolcro, connessi alla questione dello stato delle anime nell'aldilà ${ }^{33}$.

\section{Commentare l'Eneide alla luce della Commedia}

L'ultimo aspetto su cui vorrei richiamare l'attenzione è la modalità con cui, accanto all'utilizzo costante dell'Eneide, il predicatore si avvale spesso anche della Commedia. Se il primo sermone mostra come i versi danteschi possano essere inseriti nello sviluppo catechetico, in forme non difformi da quelle riscontrabili in altri sermonari dell'epoca, in diversi casi i versi sono invece introdotti a 'commento' del racconto dell'Eneide, sfruttando gli effettivi punti di contatto tra i due poemi o costruendo efficaci nessi tra i due testi. Lo mostra bene l'inizio del sermone in cui si narra l'avviarsi di Enea verso l'Ade. I passaggi tra Eneide e Commedia sono ripetuti, cosicché il racconto - in prosa del sacrificio compiuto da Enea agli dei e del susseguente terremoto (Eneide VI, 236-258) è commentato attraverso la descrizione in versi del terremoto che fa svenire Dante alla fine di Inferno III, per poi tornare immediatamente ai versi con cui la Sibilla esorta Enea a intraprendere il cammino. La descrizione poi del notturno «iter in silvis» dell'Eneide spinge - coerentemente - a citare le due terzine di apertura della Commedia e, solo a quel punto, aggiungere i versi dell'Eneide e avviare la lettura morale di questa discesa 
agli inferi, percorsa sotto un'incerta e intermittente luce lunare e immagine della vita umana - la dantesca «nostra vita».

Nunc restat dicendi quomodo Heneas facto sacrifitio et diis invocatis venit versus infernum, unde nota quod Heneas secundum preceptum Sibille fecit sacrifitium quatuor thaurorum et unius agni et unius vacche et tunc visa est terra movere sub pedibus eorum et audite sunt voces canum sive ferarum. Unde Dantes tertio infernii:

Finito questo, la buia campagnia

tremò sì forte, che dello spavento

la mente de sudore anchora se bagna.

La terra lacrimosa dedj vento,

che balenò una luce vermiglia

la qual me vense ciascun sentimento.

Et chaddj como l'omo che sonno el piglia (Inferno III, 130-136).

Tunc Sibilla incepit clamare dicens:

«Procul, o procul este ${ }^{34}$, profani» -

conclamat vates - «totoque <ab>sistite luco» (Eneide VI, 258-259).

Et dixit Henee:

«Tuque invade viam vaginaque eripe ferrum» (Eneide VI, 260).

Nunc a vatibus dictis, intraverunt antrum ${ }^{35}$ apertum. Erat autem eis tale iter quod ipsi ibant invisa per umbras et ibant per varias ${ }^{36}$ domos Plutonis et eorum iter erat tale quale est in silvis per incertam lunam ${ }^{37}$, quando scilicet sunt nebule condempsate quando aliquando apparet aliquando non, sic videbatur illud iter, per quod iba $<n>t$, quia nec clare videbant, nec omnino in tenebris erant ${ }^{38}$. Et hic incepit Dantes suum opus. Dantes primo canto inferni:

Nel meço del camino de nostra vita

me retrovaj per una selva oscura,

ché la deritta via era smarrita.

Et quanto a dire ella era ${ }^{39}$ cosa e dura

questa <selva> selvagia aspera e forte

che nel pensiero renova la paura! (Inferno I, 1-6).

Unde dicit Virgilius:

Ibant obscuri sola $<$ sub $>$ nocte per umbras

perque domos Ditis vacuas et inania regna:

quale per incertam lunam sub luce maligna

est iter in silvis, ubi celum condidit umbram (!)

Iuppiter, et nox rebus abstulit atra colorem (Eneide VI, 268-272).

Videamus aliquid moraliter: via ista per quam fit descensus ad infernum est presens vita, qua pervenimus ad mortem $[. . .]^{40}$.

\section{Conclusione}

17 Lo studio di questo sorprendente testo è appena agli inizi e richiederà una maggiore indagine tanto dei concreti contenuti morali e religiosi sviluppati da questo predicatore, quanto delle modalità di interpretare l'Eneide - e delle eventuali fonti a cui l'autore attinge $e^{41}$ - e della finalità di uno spregiudicato utilizzo di Virgilio dal pulpito. L'effettiva possibilità di rispondere a tali questioni dipenderà anche, in non piccola parte, dal ritrovamento o meno di una copia completa del Quaresimale di Enea, visto che il manoscritto di Assisi segue solo un terzo del racconto, dall'arrivo dell'eroe troiano in Italia all'incontro con Caronte.

Si possono però fin d'ora indicare due elementi di particolare rilievo in questo testo. Primo, il quaresimale è una precoce attestazione di una nuova tipologia di sermonari 
costruiti come un unico macro-racconto. A tale altezza cronologica, l'unico caso noto è quello del Quadragesimale peregrini (ante 1420) che, seppure a un livello più sofisticato di costruzione e drammatizzazione del racconto, condivide diversi elementi con il Quadragesimale Enee - dall'idea del viaggio nell'aldilà e della sua valenza didatticocomunicativa, all'utilizzo, in proporzioni invertite, della Commedia e dell'Eneide ${ }^{42}$. Insieme, questi due testi rendono assai più mosso della vulgata il quadro della predicazione italiana nel passaggio tra Tre e Quattrocento. Non bisognerà infatti aspettare il fiorire e l'imporsi della predicazione di Bernardino da Siena nel terzo decennio del Quattrocento per registrare significative novità e, cosa ancora più importante, un'effettiva ricerca di nuove forme di comunicazione religiosa. Si può anzi dire che lo sforzo del frate senese e della sua scuola contribuì a una dinamica già in atto $^{43}$.

Il secondo elemento che emerge con chiarezza è come questo testo presenti un livello di utilizzo della letteratura classica dal pulpito che non sembra avere precedenti per estensione e per valore strutturante. La stessa idea di predicare seguendo una lectura continua di un testo non biblico è eccezionale, trovando paralleli solo nell'utilizzo - o meglio 'riscrittura' - della Commedia nel Quadragesimale peregrini e, quasi un secolo dopo, nelle prediche di Johannes Geiler von Kaysersberg sul Narrenschiff di Sebastian Brant $^{44}$. In questi casi si sfruttavano però testi dall'impianto esplicitamente cristiano, le cui finalità erano conformi (almeno in parte) a quelle della predicazione. Se Virgilio, lungo tutto il medioevo, era stato sentito come autore particolarmente vicino al mondo cristiano, il suo sistematico utilizzo dal pulpito richiedeva una mediazione non scontata ${ }^{45}$. Proprio a inizio Quattrocento, ad esempio, un predicatore di spicco come Vicent Ferrer criticò a più riprese l'uso di Virgilio nelle prediche ${ }^{46}$, mostrando così che la prassi era diffusa e insieme osteggiata. Resta inoltre da valutare quanto giochi l'influenza del primo umanesimo nella scelta di presentare dal pulpito una lettura allegorica di Virgilio in chiave sia morale che teologica. Da un lato l'approccio all'Eneide è finalizzato a un programma cristiano, secondo la prassi medievale di recuperare il mondo classico leggendolo alla luce della fede, come a un livello impareggiabile aveva in definitiva fatto lo stesso Dante ${ }^{47}$. Dall'altro lato, non si può non pensare al vivace, a tratti incandescente, dibattito sull'utilizzo o meno dei testi dei poeti 'pagani' nella paideia cristiana e nella predicazione stessa che si svolgeva in Italia proprio nei decenni a cavallo tra Tre e Quattrocento: basti menzionare l'emblematico Lucula noctis di Giovanni Dominici, scritto nel 1405, o le discussioni a seguito della rimozione della statua di Virgilio a Mantova nel $1397^{48}$. Rispetto a tale dibattito, consapevolmente o meno, il Quadragesimale Enee rappresenta una fattiva presa di posizione sulla possibilità, utilità e forse attrattiva di reducere Virgilio al Vangelo, facendo dell'Eneide se non un libro sacro, un prezioso strumento catechetico. 


\section{BIBLIOGRAFIA}

BARBALARGA Donatella, Del Torso Iacopino, in Dizionario biografico degli italiani, Roma, Istituto della Enciclopedia Italiana, 1960-, vol. XXXVIII, 1990, pp. 305-306.

BASWELL Christopher, Virgil in Medieval England: Figuring the Aeneid from the Twelfth Century to Chaucer, Cambridge, Cambridge University Press, 1995.

BeLlomo Saverio, «Or sè tu quel Virgilio?»: Ma quale Virgilio?, «L'Alighieri», vol. XLVII, 2016, pp. 5-18.

BÉRIOU Nicole, Les instruments de musique dans l'imaginaire des prédicateurs, in Ead., Religion et communication : un autre regard sur la prédication au Moyen Âge, Genève, Droz, 2018, pp. 123-145.

Bernardo Silvestre, Commento all'Eneide, a cura di B. Basile, Roma, Carocci, 2008.

BöHME Hartmut et al. (a cura di), Transformation. Ein Konzept zur Erforschung kulturellen Wandels, München, Wilhelm Fink, 2011.

CABY Cécile, Oltre l'Umanesimo religioso: umanisti e Chiesa nel Quattrocento, in P. Messa, A. E.

Scandella e M. Sensi (a cura di), Cultura e desiderio di Dio. L'Umanesimo e le Clarisse dell'Osservanza, Assisi, Porziuncola, 2009, pp. 15-33.

CABY Cécile e DEssì Rosa Maria (a cura di), Humanistes, clercs et laïcs dans l'Italie du XIII ${ }^{e}$ au début du XVI siècle, Turnhout, Brepols, 2012.

CENCI Cesare, Bibliotheca manuscripta ad sacrum conventum Assisiensem, Assisi, Editrice Francescana, 1981.

DELCORNo Carlo, L'Osservanza francescana e il rinnovamento della predicazione, in I frati osservanti e la società in Italia nel secolo XV, Atti del XL Convevno internazionale (Assisi-Perugia, 11-13 ottobre 2012), Spoleto, CISAM, 2013, pp. 3-54.

DelCoRno Pietro, "Christ and the soul are like Pyramus and Thisbe": An Ovidian Story in FifteenthCentury Sermons, «Medieval Sermon Studies», vol. LX, 2016, pp. 37-60.

DELCORNo Pietro, «Et ista sunt scripta Dantis»: predicare la Commedia in Quaresima, in P. Delcorno, E. Lombardo e L. Tromboni (a cura di), I sermoni quaresimali: digiuno del corpo, banchetto dell'anima, «Memorie Domenicane», vol. XLVIII, 2017, pp. 125-143.

DELCORNo Pietro, In the Mirror of the Prodigal Son: The Pastoral Uses of a Biblical Narrative (c. 1200-1550), Leiden, Brill, 2017.

DELCORNO Pietro, Un pellegrinaggio nell'inferno dantesco: il Quadragesimale peregrini cum angelo, in G. Strinna e G. Mascherpa (a cura di), Predicatori, mercanti, pellegrini. L'Occidente medievale e lo sguardo letterario sull'Altro, Mantova, Universitas Studiorum, 2018, pp. 219-250.

DELCORNo Pietro, Quaresimali 'visibili': il serafino, il guerriero, il pellegrino, «Studi medievali», III ser., vol. LXI, 2019, in corso di stampa.

DELCORNO Pietro, LOMBARDo Eleonora e TROMBONI Lorenza (a cura di), I sermoni quaresimali: digiuno del corpo, banchetto dell'anima, «Memorie Domenicane», vol. XLVIII, 2017

Fulgenzio Fabio P., Commento all'Eneide, a cura di F. Rosa, Trento, Luni, 1997.

GIORDANO DA PISA, Quaresimale fiorentino 1305-1306, a cura di C. Delcorno, Firenze, Sansoni, 1974. 
GIOVÈ Nicoletta e ZAMPONI Stefano, Manoscritti in volgare nei conventi dei frati Minori: testi, tipologie librarie, scritture (secoli XIII-XIV), in Francescanesimo in volgare (secoli XIII-XIV), Atti del XXIV Convegno internazionale (Assisi, 17-19 ottobre 1996), Spoleto, CISAM, 1997, pp. 301-336.

GómEZ Francesco J., Dante e Pietro Alighieri nell'opera teologia del minorita catalano Joan Pasqual, «Studi danteschi», vol. LXXX, 2015, pp. 243-292.

HOWARD Peter, Making a City and Citizens: The 'Fruit' of Preaching in Renaissance Florence, in A. Brown e J. Dymolyn (a cura di), Medieval Urban Culture, Turnhout, Brepols, 2017, pp. 59-73.

ITALIA Sebastiano, Il Virgilio medievale: tra filologia, filosofia e leggenda, Acireale, Bonanno, 2012.

LORD Mary Louise, A Commentary on Aeneid 6: Ciones de Magnali, not Nicholas Trevet, «Medievalia et humanistica», n.s., vol. XV, 1987, pp. 147-160.

MALDINA Nicolò, Predicare l'aldilà. Osservazioni sul Quaresimale di Giordano da Pisa (Firenze, 1305-1306), «Italianistica», vol. XLIII, nº 1, 2014, pp. 11-29.

MALDINA Nicolò, In pro del mondo: Dante, la predicazione e i generi della letteratura religiosa medievale, Roma, Salerno, 2017.

MÉsoniat Claudio, Poetica theologia. La Lucula Noctis di Giovanni Dominici e le dispute letterarie tra '300 e'400, Roma, Edizioni di Storia e Letteratura, 1984.

PADOAN Giorgio, Il pio Enea, l'empio Ulisse. Tradizione classica e intendimento medievale in Dante, Ravenna, Longo, 1977.

PEgoRETTI Anna, Immaginare la veste di un angelo: il caso di Purg. IX, 115-16, «L'Alighieri», vol. XXVII, 2006, pp. 141-150.

RAMIRES Giuseppe, Commento di Servio al libro VI dell'Eneide: citazioni filosofiche e memoria di Dante, in «Bollettino di italianistica», n.s., vol. VII, $\mathrm{n}^{\circ} 2,2010$, pp. 20-34.

RELTGEN-TALLON Anne, L'observance dominicaine et son opposition à l'humanisme. L'exemple de Jean Dominici, in P. Gilli (a cura di), Humanisme et Église en Italie et en France méridionale ( $X v^{e}$ siècle-milieu du XVI ${ }^{e}$ siècle), Roma, École française de Rome, 2004, pp. 43-62.

SIRI Francesco, I classici e la sapienza antica nella predicazione di Alano di Lilla, in A. Palazzo (a cura di), L'antichità classica nel pensiero medievale, Turnhout, Brepols, 2011, pp. 149-170.

VECCHIO Silvana, Le prediche e l'istruzione religiosa, in La predicazione dei frati dalla metà del '200 alla fine del '300, Atti del XXII Convegno della Società internazionale di studi francescani (Assisi, 13-15 ottobre 1994), Spoleto, CISAM, 1995, pp. 301-335.

VIRGILIO, Opera, a cura di G. Calfurnio, Vicenza, Leonardus Achates, 1479.

VISANI Oriana, Citazioni di poeti nei sermonari medievali, in G. Auzzas, G. Baffetti e C. Delcorno (a cura di), Letteratura in forma di sermone: i rapporti tra predicazione e letteratura nei secoli XIII-XVI, Firenze, Olschki, 2003, pp. 123-146.

VOLTMER Rita, "Preaching during the Holy Week Is Like Being Killed on the Wheel": The Design, Performance, and Recording of Johannes Geiler of Kaysersberg's Lenten Sermons, in P. Delcorno, E. Lombardo e L. Tromboni (a cura di), I sermoni quaresimali: digiuno del corpo, banchetto dell'anima, «Memorie Domenicane», vol. XLVIII, 2017, pp. 277-291.

WALSH Peter G. et al. (a cura di), One Hundred Latin Hymns, Cambridge, MA, Harvard University Press, 2012. 
WeNZEL Siegfried, The Classics in Late-Medieval Preaching, in A. Welkenhuysen, H. Braet e W. Verbeke (a cura di), Mediaeval Antiquity, Leuven, Leuven University Press, 1995.

\section{ALLEGATO}

\section{Sermone del Mercoledì delle Ceneri}

[f. 105r] <U>nge caput tuum et faciem tuam lava, Mat. $6^{\circ}$ [6.17]. In ista sancta quadragesima intendo predicare alica que erunt delectantia auditum et tamen dolorem incutientia et timorem. Et ideo hoc, quia volo predicare infernum secundum Virgilium, qui in $6^{\circ}$ Heneydos posuit Eneam ad ipsum infernum descendisse et ibi horrendas penas ac monstra peccatorum vidisse. Illum ergo descensum inferni et que in ipso continentur reducam ad Evangelum, gratia dei previa, et hoc faciam subcinto sermone, deinde dicam ystoriam Evangelii iusta materiam ipsius Evangelii, unde colligam fructum quo mediante ad fructum ligni vite pervenire valeamus. Et ideo, si bene advertitis, ante Evangelium predicam que effugere debemus et timere, et post Evangelium que ad vitam eternam trahunt subiungam.

Et ideo pro principio hodierne predicationis debetis scire quod postquam Eneas pervenit ad Ytalyam, invenit Sybillam Cumeham (!), que iusta templum Apollinis habitabat serviens illi deo die ac nocte ${ }^{49}$. Et remanente Enea in templum, eius sotius Achates ivit ad Sibillam intimans Eneam adesse qui cum ea volebat loqui. Unde ipsa Sibilla, simul cum Achate venit ad templum Apollinis et invenit Eneam respicentem picturas et ipsum acriter redarguit, dicens quod non decebat ut qui gratiam a deo requirebat circha Ytaliam suum tempus admictere, sed opportebat ut deo sacrifitium offerret; quod sacrifitium fuit mactatio et interfectio septem vitullorum et septem agnorum. Et tali sacrifitio peracto, Sybilla vocavit Eneam ad alta templi, ubi erant centum foramina ex quibus dabantur responsa Sibille, unde testus Virgilii:

Deyphebe Glaucy fatur que talia regi:

«Non hoc ista sibi tempus spectacula poscit;

nunc grege de intacto septem mactare iuvencos prestiterit, totidem lectas de more bidentes».

Talibus affata Eneam, nec sacra morantur

iussa Teucros viri, vocat alta $<$ in $>$ templa sacerdos ${ }^{50}$.

Sed istam particulam volo moraliter exponi, et sic omni die facere propono, quia aliter non possem reduci ad Evangelium. Dico ergo quod iste Eneas hic tenet locum cuiuslibet nostrum, qui per tempora elapsa perdidimus tempum in vanum. Nunc ergo quia tempus adest ut ad dominum humiliter revertamur, ideo Sybilla, id est divina sapientia, consulit nobis ut deo sacrifitium offeramus septem taurorum etc. [et septem agnorum]. Ista animalia, scilicet oves, sunt quinque sensus corporis et voluntas et sensualitas. Ista enim deo offerre debemus per mortificationem ut non sequamur amplius nostros appetitus sensuales, sed modo regamur secundum rationem. Et propterea canit ecclesia: «Emendemus in [f. 105v] melius | que ignoranter peccavimus | ne forte preocupati die mortis | queramus spatium penitentie | et invenire non possimus» ${ }^{51}$. Et in ymno dicitur: «Utamur ergo partius | verbis, cibis et potibus, | sompno, iocis et artius | perstemus in custodia ${ }^{52}$. Item debemus offerre septem iuvencos, id est septem peccata mortalia, ut si usque modo fuimus superbi, nunc symus humiles, si avari nunc 
liberales ${ }^{53}$ et sic de aliis. Et tunc introducemur in templum ubi dantur responsa, id est sic poterimus perduci ad templum vite eterne. Et ad illud sacrifitium faciendum ortatur nos Christus in Evangelio hodierno dum dicit verbum propositum: Unge caput tuum et faciem tuam lava. Per caput intelligitur voluntas et per faciem spiritus exteriores etc.

In isto Evangelio, sicud et in ceteris sequentibus, volo ex multis unum colligere fructum perducens ad vitam eternam. Et quando dixi in precedentibus quod ad gratiam inpetrandam debemus deo sacrifitium offerre septem ovium et totidem taurorum, scilicet quinque sensum et voluntatem et sensualitatem, id est septem peccata mortalia, sed secundum Evangelium ista non melius offerruntur et mactantur quam per ieiunium, et ideo ieiunium primo colligemus pro nostro fructu.

Circa quod quattuor per ordinem videbimus. Primo, quis fuit auctor huius ieiunii, et dico quod fuit deus. Et tale est tres, scilicet quatuor temporum, vigiliarum et quadragesime. Primum, scilicet quatuor temporum <ieiuinium> est ut sequemur preceptum legis solvendo primictias, quia sicud quattuor sunt tempora anni, sic quater facimus quator tempora, scilicet in adventu pro yeme, in quadragesima pro vere, in pentecosten pro estate, in septembri pro autumno. Secundum ieiunium est in quadragesima. Et hoc ut solvamus decimas anni, quia a prima dominica de quadragesima usque ad pasca sunt sex ebdomate, scilicet 42 dies a quibus remotis sex dominicis quibus non ieiunamus remanent 36 qui sunt decima pars totius anni. Tertium ieiunum est in vigiliis festivitatum. Et huius ratio est: precepit deus in lege quod asscendentes ad festivitatem in Yerusalem non apparerent vacui coram domino, et ideo deferimus nos munera ieiuniorum. Horum autem ieiuniorum duo statuit ecclesia, scilicet quatuor tempora et vigilias, tertium Christus consecravit, scilicet quadragesimam.

Secundo videndum est in quo loco, quia in paradisum fuit datum, quia deus dixit: De omni ligno paradisi comedas, de ligno autem scientie boni et mali ne comedas ${ }^{54}$. Sed quia non observavit expulsus fuit de paradiso et missus in vallem miserie. Ergo via et iter rectum eundi ad celum est ieiunium, unde ieiuni potet dicere: ianua sum primam cupientibus artem $^{55}$. Unde de ista porta ait Dantes capitulo nono purgatorii:

Vidi una porta con tre grade di sotto

per gire ad essa de color diversi

et uno portiero che non facia motto.

Divoto me getay ai santi piedi, misericordia chiesi che m'aprisse, ma prima al petto tre fiate me dedi. Septe P nella fronte me scripse col ponto della spada, e «Fa che lavj, quando se' dentro, queste piaghe» ${ }^{56}$.

[f. 106r] Ideo antiqui pagani hoc cognoscentes et ipsi hoc modo ieiunabant, quia illis diebis quibus suis diis sacrificare volebant non comedebant nec post solis occasum. Hoc idem et greci faciunt, similiter et saraceni ieiunant usque ad celum stellatum. Et in Ystoriis Scolasticis legitur quod Adam post expulsionem volens gratiam dei recuperare incepit ieiunare ${ }^{57}$. Similiter fecit Moyses volens legem dei recipere. Similiter fecit Helyas volens ad montem dei pervenire. Similiter et beatus Franciscus volens Christi stigmatibus insigniri. Propter<ea $>$ Ambrosius diffiniens ieiunium dicit: Ieiunium est mors culpe, vita gratie, actus honestatis et porta paradisi ${ }^{58}$. In qua porta sunt due 
guardie, una a destris et alia a sinistris. Prima a destris est humilitas et habet duas cedulas. In una scribitur: «Terra terra, audi vocem domini $»^{59}$. In secunda scribitur: «Memento homo quia pulvis es et in pulverem reverteris» ${ }^{60}$. Et cum ista guardia iam fecimus legam quando sacerdos in capite nobis posuit cineres, dicens verba ipsius humilitatis: Memento quia cinis es etc. Et hoc fit duplici de causa: primo in memoria mortis, quasi dicens: «Memor esto, quia mors non tardat» ${ }^{61}$; secundo ponit illas cineres in formam crucis, quasi dicens: «Tolle crucem tuam et sequere me crucifissum in ista quadragesima $»^{62}$. Secunda guardia est a sinistris et hec est recta intentio cordis ad deum. Et hoc in suo corde assidue rogat sive orat, dicens: «Non nobis, domine, non nobis, sed nomini tuo da gloriam» $»^{63}$. Secundo intrantibus dicit: «Omnia quecumque facitis in nomine Yhesu facite (64 $^{64}$ et si sic feceritis per portam istam intrabitis».

Tertio videndum est de utilitate ieiunii, unde legimus de santa Sibilla ieiunium, primo voluisse ad iram placandam, iusta illud: Placatus est factus dominus super malignitate quam dixerat facere populo suo ${ }^{65}$. Secundo ad veniam impetrandam ut patet de David. Tertio ad penam evitandam ut patet in Ninivitis. Quarto ad munditiam retinendam ut patet de Iudith et Ester. Quinto ad ignorantiam repellendam ut in Daniele. Sexto ad gratiam obtinendam sicud Moyses et Helyas.

Sed quia deus magis adtendit ad modum in facto quam ad factum in modo magisque considerat quomodo et qualiter aliquid fit quam illud quod fit, ideo nos in Evangelio instruit qualiter ieiunare debeamus tradens quattuor leges. Quarum prima est negativa prohibens similitatem, et hoc in prima parte Evangelii: Cum ieiunate nolite. Secunda est affirmativa docens sanctitatem: Tu autem cum ieiunas, unge etc. [f. 106v] Tertia est negativa docens cavere cupiditatem: Nolite thesaurizare vobis etc. Quarta est affirmativa docens habere celestem securitatem: thesaurizate vobis etc. ${ }^{66}$.

Sed pro declaratione istius Evangelii quero illud dubium: utrum ieiunium sit ad salutem necessarium. Et videtur quod non, quia multi sunt qui numquam ieiunaverunt et tamen sunt salvati. Et multi sunt qui ieiunaverunt et tamen sunt dapnati. Ad illud dubium respondeo per Evangelium quod bonum ieiunium debet secum habere quattuor domicellas. Prima est recta intentio: cum ieiunatis. Secunda est fervens oratio: cum ieiunas unge caput tuum. Tertia cordis contritio: faciem tuam lava. Quarta est elymosinarum largitio: thesaurizate vobis theraurus in $<c e l 0>^{67}$.

\section{NOTE}

1. Testo latino in appendice. Nel trascrivere sciolgo le abbreviazioni, divido le parole, distinguo tra $u / v$, introduco o modernizzo la punteggiatura, uniformo l'uso delle maiuscole. La ricerca è stata resa possibile da una Junior Core Fellowship dell'Institute for Advanced Study della Central European University. Il suo completamento è stato favorito da un Gerda Henkel Stiftung Grant.

2. Si veda S. Vecchio, Le prediche e l'istruzione religiosa, in La predicazione dei frati dalla metà del '200 alla fine del '300, Atti del XXII Convegno della Società internazionale di studi francescani, Spoleto, CISAM, 1995, pp. 301-335 e, per un ampio quadro sulla predicazione quaresimale, P. Delcorno, E. Lombardo e L. Tromboni (a cura di), I sermoni quaresimali: digiuno del corpo, banchetto dell'anima, «Memorie Domenicane», vol. XLVIII, 2017.

3. Su questa tipologia di sermonari rimando a P. Delcorno, «Et ista sunt scripta Dantis»: predicare la Commedia in Quaresima, in I sermoni quaresimali, cit., pp. 125-143 e Id., In the Mirror of the Prodigal Son: The Pastoral Uses of a Biblical Narrative (c.1200-1550), Leiden, Brill, 2017, pp. 310-369. Sulle 
sperimentazioni tra Trecento e primo Quattrocento, si veda anche Id., Quaresimali 'visibili': il serafino, il guerriero, il pellegrino, «Studi medievali», III ser., vol. LXI, 2019, i.c.s.

4. Questa caratteristica poteva risultare funzionale all'effettiva predicazione in volgare dei sermoni, dove i versi latini di Virgilio (come le citazioni bibliche) necessitavano una mediazione linguistica.

5. Sul concetto di 'trasformazione' dei classici, si veda H. Böhme et al., Transformation. Ein Konzept zur Erforschung kulturellen Wandels, München, Wilhelm Fink, 2011, in part. pp. 39-56.

6. La tendenza invece a utilizzare $\mathrm{i}$ classici «as no more than attractive but one-dimensional springboards into the usual pool of allegories» è rilevata in S. Wenzel, The Classics in Late-Medieval Preaching, in A. Welkenhuysen, H. Braet e W. Verbeke (a cura di), Mediaeval Antiquity, Leuven, Leuven University Press, 1995, p. 130. Per un quadro - anche metodologico - sulla ricezione/ trasformazione dei classici nella predicazione tardomedievale, si veda P. Delcorno, "Christ and the soul are like Pyramus and Thisbe": An Ovidian Story in Fifteenth-Century Sermons, «Medieval Sermon Studies», vol. LX, 2016, pp. 37-60.

7. Sull'utilizzo di Virgilio e Ovidio, si veda N. Maldina, Predicare l'aldilà. Osservazioni sul Quaresimale di Giordano da Pisa (Firenze, 1305-1306), «Italianistica», vol. XLIII, nº 1, 2014, pp. 11-29 e per un loro precoce uso tra XII e XIII secolo, si veda F. Siri, I classici e la sapienza antica nella predicazione di Alano di Lilla, in A. Palazzo (a cura di), L'antichità classica nel pensiero medievale, Turnhout, Brepols, 2011, pp.149-170. L'unico caso in parte assimilabile nell'utilizzo di Eneide VI come elemento strutturale di un sermonario è il Quadragesimale peregrini, databile agli stessi anni del testo qui esaminato; cfr. P. Delcorno, «Et ista sunt scripta Dantis», cit.

8. Il ricorso a una sorta di double-coding permetteva di rivolgersi ad ascoltatori diversi attivando livelli differenti di comprensione. Per un esempio di tale tecnica nell'incorporare i classici nella predicazione, cfr. Ibid., p. 55.

9. Manoscritto cartaceo, miscellaneo composito; ff. II + 298; 225 x $155 \mathrm{~mm}$. Per una descrizione si veda C.Cenci, Bibliotheca manuscripta ad sacrum conventum Assisiensem, Assisi, Editrice Francescana, 1981, vol. I, pp. 379-380. La sezione contenente il quaresimale è composta di sei fascicoli: $\mathrm{VI}^{1}+\mathrm{III}^{1}+\mathrm{VIII}^{3}+\mathrm{IV}^{1}$. Dopo il primo fascicolo vi sono tre carte sfascicolate bianche (ff. 111r-113v); mancano tre carte dopo f. 135v, due dell'ultimo quaderno e la prima dell'ultimo fascicolo: il testo non presenta pero' salti. Al termine del penultimo quaderno vi è un richiamo (f. 129v). Nella sezione del quaresimale, lo specchio di scrittura, tracciato a piombo, è 155 x $107 \mathrm{~mm}$, a pagina intera; il numero di righe varia tra 39 e 42.

10. C. Cenci, Bibliotheca, cit., vol. I, p. 379. La notizia della presenza di citazioni dantesche è riproposta, senza aggiunte, in N. Giovè e S. Zamponi, Manoscritti in volgare nei conventi dei frati Minori: testi, tipologie librarie, scritture (secoli XIII-XIV), in Francescanesimo in volgare (secoli XIII-XIV), Atti del XXIV Convegno internazionale (Assisi, 17-19 ottobre 1996), Spoleto, CISAM, 1997, p. 311. 11. «Introductiones tertii anni lectorie Tuderti fratris Philippi de Assisio, peregrinationis tempore», Assisi, Biblioteca del Sacro Convento, Fondo Antico, ms. 517, f. 92r. Cfr. C. Cenci, Bibliotheca, cit., vol. I, pp.381-383. Il manoscritto è ricco di riferimenti al contesto storico e permette di seguirne il dipanarsi dell'azione di un predicatore in un preciso ambito locale.

12. Sermoni di Francesco degli Abbati, Nicoluccio d'Ascoli e Servasanto da Faenza, oltre a parte dello Speculum Beatae Virginis di Corrado di Sassonia. Se non copiato, è almeno appartenuto a Filippo anche il fascicolo che contiene un ampio estratto (circa 200 versi) dell'Hercules furens di Seneca (ff. 139r-145v), chiuso da un sermone del frate (f. 146rv).

13. Lo attesta l'introduzione dell'ultimo testo del medesimo quaderno: «Hanc collationem feci in conventu Arimini dum fui ibi lector, in responsione fratris Iacobi de Tolentino existente (!) pro secondo lectore»; A, f. 180r. Nel periodo precedente, Filippo è attestato come minister a Bologna (1411-1414).

14. Il thema scelto (Me querebatis; Luca 2.49) ricorre nel Vangelo della domenica dopo l'Epifania. Il sermone include un acrostico del nome Iacobus. Non è nota la data della morte di questo 
cardinale, stretto collaboratore di Gregorio XII, ma il suo testamento venne dettato il 29 agosto 1414 a Rimini, dove fu sepolto nella chiesa degli agostiniani; D. Barbalarga, Del Torso Iacopino, DBI, vol. XXXVIII, 1990, pp. 305-306.

15. C. Cenci, Bibliotheca, cit, vol. I, p. 376 (con bibliografia).

16. Per un quadro aggiornato, con particolare riferimento al contesto italiano, si veda N. Maldina, In pro del mondo: Dante, la predicazione e i generi della letteratura religiosa medievale, Roma, Salerno, 2017, pp. 34-82, dove si sottolinea la convergenza strutturale tra questi testi e la predicazione degli ordini mendicanti.

17. Il racconto riassume Eneide VI, 1-45, con alcune varianti e senza descrivere le immagini del tempio.

18. Fulgenzio, Commento all'Eneide, a cura di F. Rosa, Trento, Luni, 1997, pp.64-65. Per il commento di Servio, mi sono avvalso dell'edizione curata da Guarino da Verona con le aggiunte di Giovanni Calfurnio, ritenendola una buona testimonianza dei materiali in circolazione nell'Italia del Quattrocento; Virgilio, Opera, a cura di G. Calfurnio, Vicenza, Leonardus Achates, 1479, f.r3r. Sul commento di Servio, rimando a G. Ramires, Commento di Servio al libro VI dell'Eneide: citazioni filosofiche e memoria di Dante, «Bollettino di italianistica», n.s., vol. VII, $\mathrm{n}^{\circ} 2$, 2010, pp. 20-34 (con bibliografia) e, di carattere più introduttivo, S. Italia, Il Virgilio medievale: tra filologia, filosofia e leggenda, Acireale, Bonanno, 2012 (fermandosi al XII secolo, risulta poco utile per la nostra analisi).

19. B. Silvestre, Commento all'Eneide, a cura di B. Basile, Roma, Carocci, 2008, pp. 108-110. Si veda anche l'ottima introduzione del curatore, ivi, pp. 7-28 e le osservazioni sulla presenza del testo in Italia tra Tre e Quattrocento in G. Padoan, Il pio Enea, l'empio Ulisse. Tradizione classica e intendimento medievale in Dante, Ravenna, Longo, 1977, pp. 207-222.

20. Si vedano le considerazioni in P. Howard, Making a City and Citizens: The 'Fruit' of Preaching in Renaissance Florence, in A. Brown e J. Dymolyn (a cura di), Medieval Urban Culture, Turnhout, Brepols, 2017, p. 63.

21. La lista di esempi dell'utilitas del digiuno poteva, evidentemente, essere trattata con una certa ampiezza, così come la parte conclusiva sulle domicelloe che devono accompagnare il digiuno.

22. Sulla tendenza di citare Dante a conferma di quanto esposto, ma senza commentare i suoi versi, si veda O. Visani, Citazioni di poeti nei sermonari medievali, in G. Auzzas, G. Baffetti e C. Delcorno (a cura di), Letteratura in forma di sermone: i rapporti tra predicazione e letteratura nei secoli XIII-XVI, Firenze, Olschki, 2003, pp.123-146. Diverso in questo senso è il caso del Quadragesimale peregrini, cfr. P. Delcorno, Un pellegrinaggio nell'inferno dantesco: il Quadragesimale peregrini cum angelo, in G. Strinna e G. Mascherpa (a cura di), Predicatori, mercanti, pellegrini. L'Occidente medievale e lo sguardo letterario sull'Altro, Mantova, Universitas Studiorum, 2018, pp. 219-250.

23. Si può pensare a una sorta di double-coding: chi conosceva il poema dantesco poteva cogliere $\mathrm{e}$ apprezzare l'interazione tra le due citazioni a un livello di maggiore complessità.

24. Il richiamo alle stimmate è un chiaro indizio della paternità minoritica del sermonario - a meno di considerarlo un'aggiunta del copista.

25. Nell'associazione del guardiano con il binomio terra/cenere, oltre all'evidente rimando alla liturgia del giorno, può dipendere anche da un riferimento all'abito dell'angelo in Purgatorio IX, 115-116, tanto più considerata la presenza di una tradizione interpretativa, inaugurata dall'Ottimo, che legava i colori della sua veste non solo al tema dell'umiltà, ma proprio a Genesi 3.19 (citato qui nel sermone), come nota A. Pegoretti, Immaginare la veste di un angelo: il caso di Purg. IX, 115-16, «L'Alighieri», vol. XXVII, 2006, p. 143.

26. A, f. 121v. I versi citati sono Eneide VI, 149-154, seguiti - con nesso logico precario - da Inferno I, 112-120.

27. «Et fructus iste erit considerare statum merchatorum qui de templo eiciuntur. Ponuntur tamen in isto Evangelio duo status merchatorum, unus habet pedem claudum (cfr. Matteo 21.14), 
secundus vero status merchatorum qui habent curam pastoralem. Et quia de isto statu cum sobrietate debemus loqui, ideo solum de primo statu intendo loqui, quem Christus de templo expulit cum flagellis; sed sacerdotibus cum mansuetudine respondit»; A, f. 122r. La sottolineatura riguardo alla necessaria prudenza nel trattare dei peccati del clero - oltre a riflettere una precisa prassi pastorale che riservava il tema ai sermoni ad clerum - evidenzia come il ciclo sia concepito per un'effettiva predicazione ad populum.

28. A, ff. $122 \mathrm{v}-123 \mathrm{r}$.

29. Bernardo Silvestre, Commento, cit. pp. 148-151, dove si riprende e amplia Fulgenzio.

30. A: neptum - forse errore di lettura del copista.

31. A, f. 123r. L'ultimo versetto ripete il thema del sermone.

32. Si veda il ricco quadro in N. Bériou, Les instruments de musique dans l'imaginaire des prédicateurs, in Ead., Religion et communication: un autre regard sur la prédication au Moyen Âge, Genève, Droz, 2018.

33. $A$, ff. $123 \mathrm{r}-124 \mathrm{v}$.

34. A: hoste.

35. Passo oscuro; sulla base di Eneide VI, 262 che parla di antro aperto, correggo ad sensum il poco comprensibile: amenum.

36. Forse in origine vacuas, come in Virgilio.

37. A: incerta limina. Ritengo più probabile l'uso dell'espressione virgiliana, in quanto la luna è il soggetto della successiva frase «quando aliquando apparet aliquando non", come ripete successivamente anche la spiegazione allegorica.

38. Il tema della presenza di una minima luce forse deriva da Servio; G. Ramires, Commento, cit., p. 23.

39. A: ellear (?).

40. A, f. 129r.

41. Andrà ad esempio investigato il commento trecentesco di Zono de' Magnali; cfr. M. L. Lord, A Commentary on Aeneid 6: Ciones de Magnali, not Nicholas Trevet, «Medievalia et humanistica», n.s., vol. XV, 1987, pp. 147-160. Tra i canali d'accesso all'interpretazione di Virgilio da parte di questo predicatore non va escluso un influsso dello stesso Dante - abbondantemente citato - e forse anche dei commenti alla Commedia.

42. Sul Quadragesimale peregrini, vedi note 7 e 22.

43. Un quadro aggiornato in C.Delcorno, L'Osservanza francescana e il rinnovamento della predicazione, in Ifrati osservanti e la società in Italia nel secoloXV, Atti del XL Convevno internazionale (Assisi-Perugia, 11-13 ottobre 2012), Spoleto, CISAM, 2013, pp. 3-54.

44. Su Geiler veda R. Voltmer, "Preaching during the Holy Week Is Like Being Killed on the Wheel": The Design, Performance, and Recording of Johannes Geiler of Kaysersberg's Lenten Sermons, in I sermoni quaresimali, cit., pp. 277-291.

45. Un parallelo rilevante, pur geograficamente distante, è il cosiddetto Norwich Commentary (post 1381) studiato in C. Baswell, Virgil in Medieval England: Figuring the Aeneid from the Twelfth Century to Chaucer, Cambridge, Cambridge University Press, 1995, pp. 136-164. In tale testo, molte delle glosse all'Eneide di tipo morale o cristologico sono orientate a diventare exempla per la predicazione (p. 152) e «the whole Virginian Underworld undergoes a domesticating transformation [...] being seen in light of a Christian purgatory and hell» (p.156). Giustamente Baswell sottolinea l'influsso dell'Ovide moralisé su tali procedimenti.

46. Sui riferimenti ostili al «bon Virgili que jau enmig de infern» - ma anche a Dante - nelle prediche tenute da Ferrer tra 1412 e 1413, si veda F. J. Gómez, Dante e Pietro Alighieri nell'opera teologia del minorita catalano Joan Pasqual, «Studi danteschi», vol. LXXX, 2015, p. 269.

47. In aggiunta alla bibliografia già citata, si veda S. Bellomo, "Or sè tu quel Virgilio?»: Ma quale Virgilio?, «L'Alighieri», vol. XLVII, 2016, pp. 5-18. 
48. Si veda C. Mésoniat, Poetica theologia. La Lucula Noctis di Giovanni Dominici e le dispute letterarie tra'300 e'400, Roma, Edizioni di Storia e Letteratura, 1984 (p. 38 sull'episodio mantovano) e A. Reltgen-Tallon, L'observance dominicaine et son opposition à l'humanisme. L'exemple de Jean Dominici, in P. Gilli (a cura di), Humanisme et Église en Italie et en France méridionale (XVe siècle - milieu du XVI $I^{e}$ siècle), Roma, École française de Rome, 2004, pp. 43-62. Un convincente invito a superare la semplicistica e largamente inadeguata opposizione storiografica tra «esprit médiéval et esprit moderne, religiosité et laïcité» e a cogliere invece l'incessante interscambio tra chierici e laici è avanzato in C. Caby e R. M. Dessì (a cura di), Humanistes, clercs et laïcs dans l'Italie du XIII au début du $\mathrm{XVI}^{e}$ siècle, Turnhout, Brepols, 2012 (cit. p. 12). Si veda anche l'utile quadro fornito in C. Caby, Oltre l'Umanesimo religioso: umanisti e Chiesa nel Quattrocento, in P. Messa, A. E. Scandella e M. Sensi (a cura di), Cultura e desiderio di Dio. L'Umanesimo e le Clarisse dell'Osservanza, Assisi, Porziuncola, 2009, pp. 5-33. Giustamente è stato notato che un umanista come Coluccio Salutati ( $\uparrow 1406)$ non solo conosceva l'interpretazione allegorica del Silvestre, ma mostrò di apprezzarla; G. Padoan, Il pio Enea, cit., pp. 217-218.

49. La descrizione echeggia quella della profetessa Anna in Luca 2.37.

50. Eneide VI, 36-41. Qui e altrove il copista non pare particolarmente attento nel trascrivere i versi di Virgilio. Correggo come evidenti errori: Glancy, grage, bibentes, teneros.

51. Antifona del Mercoledì delle Ceneri.

52. Terza strofa dell'inno di Quaresima Ex more docti mystico servemus; cfr. P. G. Walsh et al., One Hundred Latin Hymns, Cambridge, MA, Harvard University Press, 2012, pp. 172-175 e 447-448.

53. A: civerales.

54. Genesi 2.16-17.

55. Intendendo per «prima arte» la penitenza; cfr. Giordano da Pisa, Quaresimale fiorentino 1305-1306, a cura di C. Delcorno, Firenze, Sansoni, 1974, pp. 74-75.

56. Purgatorio IX, 76-78 e 109-114.

57. Il racconto non figura nella Historia scolastica.

58. Ripresa assai libera di Ambrogio, De Elia et ieiunio, III.4, PL 14, col. 733.

59. Geremia 22.19.

60. Cfr. Genesi 3.19.

61. Siracide 14.12 .

62. Cfr. Matteo 16.24.

63. Salmo 113.9

64. Cfr. Colossesi 3.17.

65. Esodo 32.14

66. Citazioni da Matteo 6.16-20, pericope del giorno.

67. Vedi nota 65.

\section{RIASSUNTI}

L'articolo costituisce la prima esplorazione di quello che proponiamo di chiamare Quaresimale di Enea, un testo conservato in forma incompleta in Assisi, Biblioteca del Sacro Convento, Fondo Antico, ms. 557. Il quaresimale costituisce una precoce attestazione di una nuova tipologia di sermonari costruiti come un unico macro-racconto. I suoi sermoni seguono infatti passo a passo la discesa agli inferi di Enea, rileggendo il racconto di Virgilio in chiave allegorico-morale, così da 
ricondurlo (reducere) al Vangelo. Rendendo strutturale il riferimento a Virgilio nella predicazione dell'aldilà cristiano, agli ascoltatori era presentata un'Eneide infernale, didattica, ad usum pulpiti, ulteriormente arricchita dall'intreccio con i rimandi alla Commedia di Dante. Lo studio di questo sorprendente quaresimale virgiliano contribuisce così a illuminare sia le sperimentazioni in atto nella comunicazione religiosa tra fine Trecento e inizio Quattrocento sia i concomitanti dibattiti sull'utilizzo della poesia 'pagana' nella paideia cristiana.

Cet article ouvre l'étude de ce que nous nous proposons de désigner comme le Sermonnaire pour le Carême d'Énée, un texte conservé sous une forme incomplète à Assise (Biblioteca del Sacro Convento, Fondo Antico, ms. 557). Il s'agit là du témoignage précoce d'une nouvelle typologie de sermonnaires, construits comme un unique macro-récit. Les sermons y suivent en effet pas à pas la descente aux Enfers d'Énée, tout en envisageant le récit virgilien selon une clé de lecture allégorico-morale, dans le but de le raccorder (reducere) à l'Évangile. La référence à Virgile étant donnée pour structurelle dans la prédication de l'au-delà chrétien, une Énéide infernale et didactique, ad usum pulpiti, était présentée aux auditeurs, que venaient ensuite enrichir des renvois à la Divine Comédie de Dante. L'étude de cet étonnant recueil virgilien contribue à éclairer les expérimentations observées dans la communication religieuse au tournant des $\mathrm{XIV}^{\mathrm{e}}$ et $\mathrm{XV}^{\mathrm{e}}$ siècles, mais aussi les débats sur l'opportunité d'utiliser la poésie "païenne " dans la paideia chrétienne.

The article represents a first exploration of what can be defined the Lenten sermon collection of Aeneas, a text held in an incomplete form in Assisi, Biblioteca del Sacro Convento, Fondo Antico, ms. 557. The Lenten cycle is an early testimony of a new typology of sermon collection, which was framed as a macro-narrative. The Virgilian sermon collection follows closely the description of Aeneas' descent to the Underworld by reinterpreting Virgil's account according to a moralallegorical exegesis, which allows to align it (reducere) to the Gospel. By using extensively the reference to Virgil in preaching the Christian afterlife, the preacher presented his audience with a hellish, didactic Aeneid, apt to the pulpit and further enriched by references to Dante's Commedia. The study of this exceptional text, therefore, sheds light on the innovations in religious communication developed between late fourteenth and early fifteenth century as well as on the parallel debates about the use of 'pagan' poets in Christian paideia.

\section{INDICE}

Parole chiave : predicazione medievale, Virgilio, Eneide, allegoria, ricezione classici, Commedia Mots-clés : prédication médiévale, Virgile, Énéide, allégorie, réception des classiques, Divine Comédie

Keywords : medieval preaching, Virgil, Aeneid, allegory, reception of classics, Commedia

\section{AUTORE}

\section{PIETRO DELCORNO}

Radboud University Nijmegen / University of the Witwatersrand 